\title{
DYNAMIC SINGLE-PHOTON EMISSION COMPUTED TOMOGRAPHY DATA ANALYSIS: CAPABILITIES FOR DETERMINING FUNCTIONAL SIGNIFICANCE OF CORONARY ARTERY ATHEROSCLEROSIS
}

\author{
Andrew Mochula ${ }^{1 \mathrm{a}}$, Konstantin Zavadovsky $^{1,2}$, Sergey Andreev $^{1}$, Yury Lishmanov ${ }^{1,2}$ \\ ${ }^{1}$ Federal State Budgetary Scientific Institution "Research Institute for Cardiology", 634012, Tomsk, \\ Russia \\ ${ }^{2}$ National Research Tomsk Polytechnic University, 634050, Tomsk, Russia
}

\begin{abstract}
The aim of the study was to develop the method for collecting and processing scintigraphic data to determine myocardial flow reserve by using gamma-camera with a new type detector based on Cadmium Zinc Telluride (CZT). Sixteen coronary artery disease patients and nine healthy volunteers received cardiac dynamic single-photon emission computed tomography with technetium $99 \mathrm{~m}$ methoxyisobutylisonitrile $\left({ }^{99 \mathrm{~m}} \mathrm{Tc}-\mathrm{MIBI}\right)$ at rest and during pharmacologic stress test. Data processing involved a formation of regions of interest from the left ventricular (LV) cavity and myocardial walls to build activity-time curves. Myocardial flow reserve index was determined as a quotient of two ratios of mean myocardial counts to area under a curve peak from LV cavity during stress test and at rest. Mean values of myocardial flow reserve index were $1.86(1.59 ; 2.2)$ in group of healthy volunteers and $1.39(1.12 ; 1.69)$ in patients with multivessel coronary artery disease. When the value of this index was less than 1.77, the method allowed for identification of multivessel disease with sensitivity and specificity of $81.8 \%$ and $66.7 \%$, respectively. Standard myocardial perfusion scintigraphy in combination with the method of myocardial flow reserve index determination allows for increasing diagnostic significance of the scintigraphic approach for assessment of coronary microcirculation lesions in multivessel coronary artery disease.
\end{abstract}

\section{Introduction}

Coronary artery disease is currently number one cause of disability and mortality from cardiovascular diseases. According to estimates of the World Health Organization, 7.4 million people had died of this pathology worldwide during the period from 2002 to 2012.

Revascularization procedure is currently considered the efficacious method for treatment of this pathology. This method consists in recovery of coronary blood flow

${ }^{a}$ Corresponding author: mochula.andrew@gmail.com 
through coronary artery bypass surgery or through stent placements in coronary arteries [1]. According to the recommendations of the European Society of Cardiology, 50-90\% coronary artery stenosis requires surgical restoration of blood flow only in case of verified ischemia which can be confirmed by determining fractional flow reserve. Stenosis is considered hemodynamically significant if the above mentioned index equals 0.8 and less [1]. An invasive nature of the procedure as well as a high cost of the supplies do not allow for widespread use of this approach in clinical practice.

Positron emission computed tomography with ${ }^{15} \mathrm{O}$-labeled water $\left(\left[{ }^{15} \mathrm{O}\right] \mathrm{H}_{2} \mathrm{O}\right)$ is considered gold standard for noninvasive assessment of myocardial flow reserve $\left(\left[{ }^{15} \mathrm{O} \mathrm{H}_{2} \mathrm{O}\right)[2,3]\right.$. However, this method requires available cyclotron and expensive diagnostic equipment in a clinic delivering these studies. All these factors along with short half-life time of ${ }^{15} \mathrm{O}(122.2 \mathrm{~s})$ also significantly limit wide distribution of this method in everyday clinical practice. According to recommendations of the European Society of Cardiologists regarding myocardial revascularization, single-photon emission computed tomography (SPECT) plays an important role in diagnosing ischemia of the myocardium with sensitivity and specificity of $90-91 \%$ and $75-84 \%$, respectively [4]. It is worth mentioning that SPECT quite often gives false-negative results in patients with hemodynamically significant multivessel coronary artery disease showing the absence of significant stress-induced perfusion defects. Literature describes this phenomenon by term "balanced ischemia" where a uniform decrease in an indicator accumulation is observed over the entire left ventricular (LV) myocardium [5, 6]. One of the ways to diagnose balanced ischemia of the myocardium in multivessel coronary artery disease consists in an assessment of myocardial flow reserve [7, 8].

With reference to the above, search for new methods for an assessment of this parameter is a relevant task of modern cardiology [9].

Up to now, most scintigraphy works in this direction were performed by using gammacameras with detectors based on sodium iodide which did not allow for acquiring dynamic data in a tomographic mode [2,8]. Implementation of SPECT with detectors based on Cadmium Zinc Telluride (CZT) allows for overcoming technical constraints and opens up new opportunities for a radionuclide determination of myocardial flow reserve.

The aim of this study was to determine the informativity of dynamic tomoscintigraphy of the myocardium in identifying multivessel coronary artery disease.

\section{Method of study}

To determine coronary reserve, we proposed to perform myocardial SPECT with technetium $99 \mathrm{~m}$ methoxyisobutylisonitrile $\left({ }^{99 \mathrm{~m}} \mathrm{Tc}-\mathrm{MIBI}\right)$ at rest and during pharmacological stress test.

\subsection{Preparation}

Patients were recommended to abstain from caffeine-containing beverages and/or medications for twelve hours before the procedure. The last meal was taken not less than two hours before the study. Immediately before the study, intravenous catheter was placed in a median cubital vein of a subject.

\subsection{Study procedure}

A patient was accommodated in a supine position on the table of gamma-camera. For correct patient positioning relative to gamma-camera detectors, low-dose computed 
tomography $(100 \mathrm{keV} ; 10 \mathrm{~mA} / \mathrm{s})$ in the anterior projection was performed. Distances from suprasternal notch and left anterior auxiliary line to the center of cardiac silhouette were measured on an acquired image and a mark was placed on patient's body. Detectors of gamma-camera were positioned over the left ventricular (LV) area based on the acquired results [10].

A scintigraphy study was performed in two stages (Figure 1). At stage 1, the passage of a bolus of radiopharmaceutical was recorded at rest. To achieve this, a bolus injection of 1 $\mathrm{mL}$ of ${ }^{99 \mathrm{~m}} \mathrm{Tc}-\mathrm{MIBI}$ in a dose of $185 \mathrm{MBq}$ was done through the intravenous peripheral catheter. Recording began $5 \mathrm{~s}$ before the injection of radiopharmaceutical. Scintigraphic images were registered in a dynamic tomographic list mode format (allowing for reconstruction of series of dynamic and static tomoscintigrams from native data sets) for $360 \mathrm{~s}$. This time was enough for registering the passage of radioactive indicator through cardiac chambers and its accumulation in the LV myocardium. Immediately after the completion of data acquisition, stage 2 began where the passage of a bolus of radiopharmaceutical was recorded during pharmacological stress test [10].

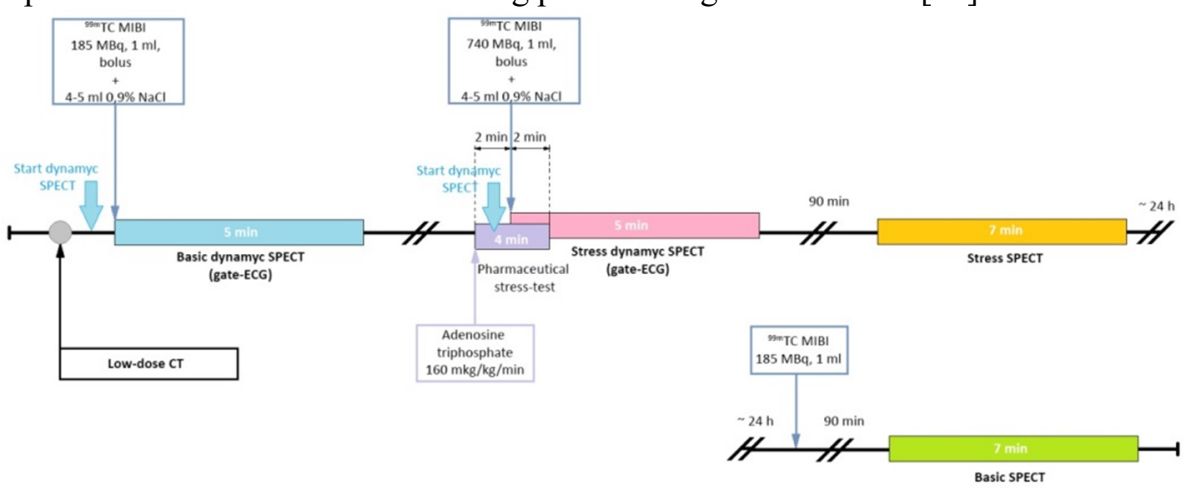

Figure 1. Scheme of cardiac dynamic SPECT for determination of myocardial flow reserve.

All scintigraphic studies were performed by using gamma-camera with CZT-based solid-state detectors (GE Discovery NM/CT 570 C) with adjustment to ${ }^{99 \mathrm{~m}}$ Tc photopeak $(140 \mathrm{keV})$.

Stress test with adenosine triphosphate (ATP) was performed according to standard protocol [3]. Adenosine triphosphate dose $(140 \mu \mathrm{g} / \mathrm{kg} / \mathrm{min})$ was calculated individually depending on patient's body weight. Adenosine triphosphate was administered with a mechanical infusion pump according to 4-min protocol (2-min ATP infusions before and after the injection of radiopharmaceutical). At pharmacological stress test peak at the end of minute 2 of ATP infusion, patient received $1 \mathrm{~mL}$ of radiopharmaceutical ${ }^{99 \mathrm{~m}} \mathrm{Tc}-$ Technetril in a dose of $740 \mathrm{MBq}$. After that, the study continued according to the protocol identical to the one specified above. A fourfold increase in a dose of the indicator at stage 2 was necessary for leveling blood pool radioactivity after the preceding study.

\subsection{Image procession and myocardial flow reserve assessment}

Two series of axial images were formed from primary data by using dedicated software (GE Alcyone: Dynamic Analysis Tool). Series 1 comprised group of 64 dynamic tomoscintigrams with each frame duration of 2.2. s representative of the passage of a bolus of radiopharmaceutical through the LV cavity and through the LV myocardium from second 1 to second 150 of the study; series 2 comprised static tomoscintigrams from second 150 to second 360 representative of the accumulation of radiopharmaceutical in the LV walls. 
The next stage of data processing consisted in building the activity-time curves used for myocardial flow reserve calculation. To achieve this, regions of interest from the LV cavity and the LV walls were formed on the series of static frames; activity-time plots were built based on the dynamic images for each region of interest: anterior, posterior, lateral walls, septum, and apex of the left ventricle. Based on the obtained plots, mean value of counts from each region of interest in the left ventricle as well as area under curve characterizing the passage of a bolus of radiopharmaceutical through LV cavity were determined. These calculations were performed twice: for study at rest and for study during pharmacological stress test. Myocardial flow reserve index (MFRI) was determined separately for each LV wall as follows:

$$
M F R I=\frac{C_{m s}(t) / \int_{0}^{t} c_{b s}(\tau) d t}{C_{m b}(t) / \int_{0}^{t} c_{b b}(\tau) d t},
$$

where: MFRI is myocardial flow reserve index; $\mathrm{C}_{m s}$ is number of counts from the LV myocardium during stress test; $C_{b s}$ is number of counts from the LV cavity during stress test; $\mathrm{C}_{m b}$ is number of counts from the LV myocardium at rest; $C_{b b}$ is number of counts from the LV cavity at rest.

\subsection{Method approbation}

A total of 16 ischemic heart disease patients aged $67 \pm 5$ year (13 men, 3 women) with multivessel coronary artery disease diagnosed based on results of invasive coronary angiography were examined with this method. Comparison group comprised nine healthy volunteers aged $25 \pm 4$ years ( 7 men, 2 women).

Procedure of myocardial flow reserve assessment was approved by the local ethics committee of the authors' institute. All patients were informed in a written format regarding the goal and potential risks of the study and gave informed consent to perform it.

\subsection{Statistical analysis}

Statistical processing of the results was performed with SPSS 18.0 and MedCalc 12.1.14.0 software. Obtained data were not normally distributed (based on Kolmogorov-Smirnov criterion) so data were presented as median and quartiles $\left(\mathrm{Me}\left(\mathrm{Q}_{25} ; \mathrm{Q}_{75}\right)\right)$. Significance of intergroup differences was assessed based on nonparametric Mann-Whitney test. To determine differential boundary (cut-off value) between study values in independent samples, ROC-analysis with building ROC-curves was performed. Values were considered statistically significant when $p$ was $<0.05$.

\section{Results}

Based on performed analysis, mean LV MFRI in patients with three-vessel coronary artery disease significantly differed from the corresponding value in healthy volunteers group $(P<$ $0.05)$ : 1.39 (1.12; 1.69) and $1.86(1.59 ; 2.2)$, respectively.

Statistically significant decreases in MFRI values in group of patients with multivessel disease compared with the corresponding values in individuals free of coronary atherosclerosis were also found based on analysis of MFRI in separate regions of the left ventricle (Table 1). 
Table 1. Regional values of myocardial flow reserve.

\begin{tabular}{|c|c|c|c|}
\hline \multirow[b]{2}{*}{ LV regions } & \multicolumn{3}{|c|}{$\begin{array}{l}\text { Regional values of myocardial flow reserve } \\
\operatorname{Me}\left(\mathrm{Q}_{25}-\mathrm{Q}_{75}\right)\end{array}$} \\
\hline & $\begin{array}{l}\text { Group of patients with } \\
\text { multivessel coronary } \\
\text { artery disease }\end{array}$ & $\begin{array}{c}\text { Group of healthy } \\
\text { volunteers }\end{array}$ & $\begin{array}{l}p \text {-level (Mann- } \\
\text { Whitney) }\end{array}$ \\
\hline Apex & $1.3(1.0-1.76)$ & $1.83(1.65-2.04)$ & 0.03 \\
\hline Lateral wall & $1.54(1.12-1.73)$ & $2.13(1.42-2.31)$ & 0.01 \\
\hline Anterior wall & $1.27(1.0-1.7)$ & $1.65(1.19-1.98)$ & 0.02 \\
\hline Septum & $1.25(0.93-1.77)$ & $2.1(1.76-2.2)$ & 0.01 \\
\hline
\end{tabular}

The results of ROC-analysis demonstrated that MFRI value of 1.77 allowed for verification of three-vessel coronary artery disease with sensitivity of $81.8 \%$ and specificity of $66.7 \%$. The area under ROC-curve was $0.79(p<0.05)$ suggesting high confidence of the method (Figure 2).

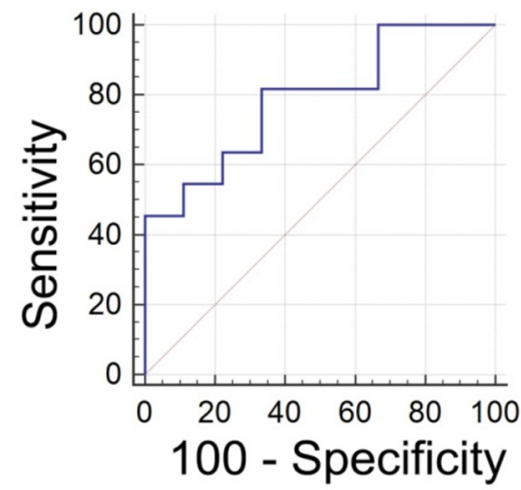

Figure 2. ROC-curve representative of diagnostic capabilities of myocardial flow reserve index in detecting multivessel coronary artery disease.

\section{Conclusion}

Search for a simple and inexpensive method for an assessment of myocardial flow reserve is currently considered one of the main directions of state-of-the-art cardiology. In the present study, for the first time, sensitivity and specificity of cardiac dynamic stress SPECT in assessment of myocardial flow reserve were determined and had values of $81.8 \%$ and $66.7 \%$, respectively. Our results rank below compared with operating characteristics of positron emission tomography with ${ }^{15} \mathrm{O}$-labeled water whose sensitivity and specificity values are $95 \%$ and $100 \%$, respectively [4]. It is caused by linear dependence of $\left[{ }^{15} \mathrm{O} \mathrm{H}_{2} \mathrm{O}\right.$ extraction fraction on myocardial flow rate as well as by higher resolution power of positron emission tomography scanners. Data obtained in our study agree with the results of other research groups who showed principal feasibility of myocardial flow reserve assessment by the methods of dynamic SPECT $[4,6,7]$ or planar scintigraphy [2] with high diagnostic accuracy values.

Therefore, implementation of the present method into clinical practice and experimental research may contribute to in-depth study of myocardial microcirculation processes and will allow for more adequate identification of treatment tactics in coronary insufficiency. 


\section{Acknowledgments}

This work was supported by Russian Science Foundation (grant \#15-15-10016).

\section{References}

[1] S. Windecker, P. Kolh, F. Alfonso and et al., Eur Heart J. 35, 2541 (2014) doi: 10.1093/eurheartj/ehu278

[2] G. Storto, AR. Sorrentino, T. Pellegrino, R. Liuzzi, M. Petretta, A. Cuocolo, Eur J Nucl Med Mol Imaging 34(8), 1156 (2007) doi: 10.1007/s00259-006-0333-x

[3] M.J. Henzlova, W.L. Duvall, A.J. Einstein, M.I. Travin, H.J. Verberne, J Nucl Cardiol 23, 606 (2016) doi: 10.1007/s12350-015-0387-x

[4] Y. Ito, C. Katoh, K. Noriyasu, Y. Kuge, H. Furuyama, K. Morita, T. Kohya, A. Kitabatake, N. Tamaki, Eur J Nucl Med Mol Imaging 30, 281 (2003)

[5] C. Liu, A.J. Sinusas, J Nucl Med. 55, 1573 (2014) doi: 10.2967/jnumed.114.144550

[6] T. Tsukamoto, Y. Ito, K. Noriyasu, K. Morita, C. Katoh, H. Okamoto, N. Tamaki, Circ J. 69,188 (2005) doi: 10.1253/circj.69.188

[7] S. Ben-Haim, V.L. Murthy, C. Breault and et al., J Nucl Med. 54, 873 (2013) doi:10.2967/jnumed.112.109652

[8] B. Hsu, F.C. Chen, T.C. Wu, W.S. Huang, P.N. Hou, C.C. Chen, G.U. Hung, Eur J Nucl Med Mol Imaging 41, 2294 (2014) doi: 10.1007/s00259-014-2881-9

[9] Yu.B. Lishmanov, K.V. Zavadovskiy, I.Yu. Efimova, N.G. Krivonogov, Zh.V. Vesnina, S.I. Sazonova and et al., The Siberian medical journal 2, 21 (2015)

[10] A.V. Mochula, K.V. Zavadovskiy, Yu.B. Lishmanov, BulletFin of Experimental Biology and Medicine 12, 845 (2015) 